\title{
Portrait du berger en figure républicaine ou comment faire entrer l'animal domestique en Révolution
}

The Portrait of a Shepard as a Republican Figure or how to introduce the Domestic Animal into the Revolution

\section{Malik Mellah}

\section{OpenEdition Journals}

Édition électronique

URL : https://journals.openedition.org/ahrf/12966

DOI : $10.4000 /$ ahrf.12966

ISSN : $1952-403 X$

Éditeur :

Armand Colin, Société des études robespierristes

Édition imprimée

Date de publication : 1 décembre 2013

Pagination : 85-110

ISBN : 978290832789

ISSN : 0003-4436

\section{Référence électronique}

Malik Mellah, « Portrait du berger en figure républicaine ou comment faire entrer l'animal domestique en Révolution », Annales historiques de la Révolution française [En ligne], 374 | octobre-décembre 2013, mis en ligne le 01 décembre 2016, consulté le 01 juillet 2021. URL : http://journals.openedition.org/ ahrf/12966 ; DOI : https://doi.org/10.4000/ahrf.12966 


\title{
PORTRAIT DU BERGER EN FIGURE RÉPUBLICAINE OU COMMENT FAIRE ENTRER L'ANIMAL DOMESTIQUE EN RÉVOLUTION
}

Malik MELLAH

\begin{abstract}
Entre la fin de l'Ancien Régime et l'Empire, l'école vétérinaire d'Alfort, ouverte en 1766, fut un des centres les plus importants d'un long travail d'amélioration des bêtes à laine en France. Si la création de l'institution répondit d'abord à une volonté de lutter contre les épizooties et à un effort en vue de fournir de bons chevaux, les moutons devinrent rapidement l'objet d'une attention particulière. Quelques vétérinaires d'Alfort se saisirent de ces préoccupations pour s'insérer dans les cadres scientifiques et nourrir l'image d'un berger savant. Dans les dernières années de la monarchie, ces bergers participèrent, aux côtés des naturalistes parisiens, d'un discours de régénération de la société. Au tournant de l'an III, leur œuvre fut mise au service d'un projet républicain de stabilisation du pays. À partir du Consulat, l'École d'Alfort fut prise dans une dynamique nouvelle, industrielle, de création d'un animal de rente. ABSTRACT
\end{abstract}

Mots-clés : Animal, agriculture, Science, Instruction,

À partir de 1789 et plus encore de l'an III, une nécessité s'impose, celle de cimenter la nation en s'ouvrant sur l'avenir et en formulant un projet de société. Pour en apprécier les fondements et la portée, des travaux récents proposent d'interroger non plus le cœur du fonctionnement de la République, dans ses aspects historiques et théoriques, mais les confins du cercle de la citoyenneté républicaine. De ces marges, les animaux peuvent former une entrée. Ceux des ménageries et des essais savants ont déjà eu leur place dans ces réflexions, interrogeant le sauvage et l'urbain, la 


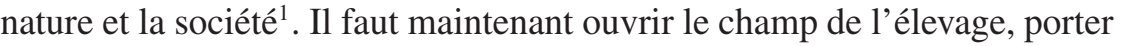
notre regard sur les bêtes à laine et formuler l'hypothèse que la place dévolue à ces animaux « utiles » induit des formes de représentation et de classement qui, au-delà de participer à l'organisation du champ des savoirs et des institutions scientifiques, construisent, en idéal et en actes, un système social, économique et politique pouvant nous aider à percevoir le changement dans l'ordre du monde entre la fin du XVIII' siècle et le début du XIX ${ }^{\mathrm{e}}$ siècle.

Dans son œuvre sur la culture équestre de l'Occident, Daniel Roche, en consacrant le premier livre au « cheval moteur », invitait à comprendre comment, dans le cadre d'une civilisation agricole dominante et dans les limites du développement préindustriel, les chevaux étaient partout, et comment reposait sur ces animaux une culture technique originale ${ }^{2}$. Pourrait-on considérer qu'il exista un «mouton moteur » ? Il n'en va pas seulement du calembour. Dès 1819, Jean-Antoine Chaptal avait, semble-t-il, bien conscience de l'effet d'entraînement que constituait spécifiquement l'élevage des bêtes à laine.

« Non seulement l'importation des mérinos a enrichi notre agriculture et notre industrie de la laine la plus fine qui fut connue en Europe ; mais encore, par le croisement des races, elle a amélioré les laines communes sur tous les points de la France. Cet avantage paraîtra inappréciable si l'on considère qu'en améliorant nos laines, on en élève la valeur, et on augmente le poids des toisons de près de moitié.

Mais ce serait mal connaître le prix que nous devons attacher à l'introduction des mérinos que de le borner à l'amélioration de nos laines : son influence sur l'agriculture est peut-être plus importante encore : les riches propriétaires se sont d'abord emparés de cette branche de l'industrie agricole ; ils ont fait, à l'envi, de grands sacrifices pour soigner ces animaux : partout on a construit des bergeries bien aérées où l'on a maintenu la plus grande propreté ; partout on a formé de bons bergers, et on les a

(1) Éric BARATAY, « La promotion de l'animal sensible, une révolution dans la Révolution », Revue historique, 1/2012 ( ${ }^{\circ}$ 661), p. 131-153; Jean-Luc GUICHET (éd.), Usages politiques de l'animalité ; Paris, l'Harmattan, 2008. Surtout Pierre SERNA, « Droits d'humanité, droits d'animalité à la fin du $18^{\mathrm{e}}$ siècle, ou la matrice du "racisme social" en controverse », Dix- Huitième siècle, $\mathrm{n}^{\circ} 42$ 2010, p. 247-263 ; Id., « The Rights of Man and the Rights of Animality at the End of the Eighteenth Century » dans Joan LANDES, Paula Young LEE and Paul YoungQUIST (dir.), Gorgeous beast, Animal bodies in Historical perspective, Penn State Press, p 89-104, notes p 191-196.

(2) Daniel RocHE, La Culture équestre de l'Occident XVI ${ }^{e}$-XIX ${ }^{e}$ siècle. L'ombre du cheval. Tome Premier le cheval moteur, Paris, Fayard, 2008 ; Id., « Les chevaux au $18^{\mathrm{e}}$ siècle », Dix-Huitième siècle, 1/2010 ( $\left.\mathrm{n}^{\circ} 42\right)$, p. 232-246. 
instruits de tout ce qui peut intéresser la conduite, la santé et les maladies des bêtes à laine ; partout on a multiplié les prairies artificielles pour leur assurer une nourriture saine et abondante. Ainsi cette acquisition a remué tous les intérêts de l'agriculture, et a contribué à en perfectionner toutes les branches $»^{3}$.

Le mouton, animal paisible et sans défense, devait également devenir une arme dans la guerre économique que la France livrait à l'Angleterre. À la fin de l'Ancien Régime comme sous l'Empire, une double conviction était partagée qu'il fallait que cesse l'exportation de numéraire engendrée par les importations d'Angleterre et qu'il était nécessaire que les productions françaises, par leur qualité et par leurs prix, puissent concurrencer celles du puissant voisin. Chez Jean-Marie Roland de la Platière comme chez Jean-Antoine Chaptal ou l'aumônier de l'Empereur, l'abbé de Pradt, on trouvait la conscience d'une avance de l'Angleterre dans le domaine des arts mécaniques. Dans cette dynamique et dans cette guerre « industrielle », l'animal était central, car à la fois machine et main-d'œuvre, énergie et matière première ${ }^{4}$.

Autour de ces considérations, une autre lecture de la place de l'animal, plus ample, peut être construite. Il s'agit alors de construire une perspective quelque peu originale dans laquelle se croisent les histoires économiques, scientifiques, sociales, politiques ou culturelles et qui visent à montrer comment dans le changement profond de l'ordre du monde de la fin du $\mathrm{XVIII}^{\mathrm{e}}$ siècle et du début du $\mathrm{XIX}^{\mathrm{e}}$ siècle, l'animal put être tout à la fois ou successivement un moteur des transformations, une fin ou un des éléments d'un projet de stabilisation de la société. L'École vétérinaire d'Alfort semble constituer un point d'observation intéressant. Elle fut, en effet, le centre matriciel principal de l'enseignement scientifique vétérinaire, permit la constitution d'un ensemble de techniques et d'un groupe de savants et de praticiens qui contribuèrent à construire et diffuser une conscience d'abord sanitaire et médicale puis économique et sociale autour de l'animal. Parmi ces vétérinaires, professeurs ou vétérinaires, la figure du berger se détache. Ce travail à partir de l'École vétérinaire amène à interroger des

(3) M. le Comte de CHAPTAL, De l'industrie française, Tome Premier, chez Antoine- Augustin Renouard, 1819

(4) Ibidem, Encyclopédie méthodique ou par ordre de matières par une société de gens de lettres, de savants et d'artistes. Manufactures, arts et métiers par M. ROLAND DE LA PLATIÈRE, Tome premier, Paris, Panckoucke Liège, Plomteux, 1785 ; Dominique DuFOUR DEPRADT, De l'état de la culture en France et des améliorations dont elle est susceptible, Tome premier, Paris, imprimerie de Guilleminet, An X, 1802. 
scansions et des ruptures plus ou moins classiques en utilisant certains angles du point de vue animal ${ }^{5}$. Dans un premier temps nous montrerons que, dans les dernières années de l'Ancien Régime, les professionnels d'Alfort contribuèrent à une redéfinition de la place des bêtes utiles dans les savoirs naturalistes et dans l'élaboration de nouvelles approches politiques. $\mathrm{Au}$ tournant de l'an III et plus encore à mesure que l'on avance dans le Directoire, nous mettrons en évidence l'émergence d'une figure de « berger républicain » au terme d'une réorganisation de l'espace savant, faisant du vétérinaire une fonction sociale et le porteur d'un projet de stabilisation. Les évocations du développement d'une nouvelle dynamique, essentiellement industrielle, autour des bêtes à laine et des bergers ainsi que des transformations des cadres scientifiques après le coup d'État du 18 Brumaire nous serviront à éclairer l'originalité de la configuration qui nous porte à considérer certains vétérinaires formés ou ayant œuvré à Alfort, les bergers, comme de véritables personnalités républicaines.

\section{Une bergerie d'utopie}

Au moment de la création des écoles vétérinaires, d'abord à Lyon en 1762 puis à Alfort en 1766, les bêtes à laine ne constituaient ni une préoccupation majeure pour les premiers vétérinaires ni un domaine d'excellence ${ }^{6}$. Si l'on se réfère aux volumes de l'Encyclopédie méthodique traitant de cette matière, on constate d'abord un volume relativement important des pages qui leur sont consacrées et un éclatement entre quatre pôles, « histoire naturelle des animaux », « agriculture », « manufactures, arts et métiers »et «médecine ${ }^{7}$. Si on met de côté les développements de l'article « médecine vétérinaire » contenu dans un des volumes de la section médecine, aucune référence substantielle ne s'appuyait sur l'œuvre des vétérinaires d'Alfort. Les références étaient alors constituées par l'œuvre économique anglaise ou par les écrits de différents agronomes échappant au champ vétérinaire. Les bêtes à laine furent pourtant introduites à l'école vétérinaire dès les jeunes années de l'institution. Sous Henri-Léonard Bertin une première ménagerie fut constituée à partir d'animaux de diverses Seuil, 2012.

(5) Éric BARATAY, Le point de vue animal, une autre version de l'histoire, Paris, éditions du

(6) Sur l'histoire des écoles vétérinaires, en particulier sur les débuts Alcide RAILLET et Léon MOULET, Histoire de l'école d'Alfort, Paris, Asselin et Houzeau, 1908 ; Henri Hours, La lutte contre les épizooties et l'école vétérinaire de Lyon au XVIII siècle, PUF, 1957.

(7) Encyclopédie méthodique, op.cit., Histoire naturelle des animaux, tome premier, A Paris, chez Panckoucke, à Madrid, chez Jacques Thevin, 1782 ; Agriculture, Tome second par l'abbé Tessier, Paris, Panckoucke, 1791. 
espèces. Si on avait rassemblé un éventail assez large d'individus, l'effort principal portait sur les bêtes à laine. On espérait un gain par l'acclimatation des espèces étrangères telles que les moutons d'Espagne, les chèvres et les boucs «d'Angora », les vigognes et les lamas. La tentative fut un échec. Les animaux soignés par les élèves souffrirent rapidement et des individus moururent dès les premiers temps de l'expérience ${ }^{8}$. Ces bêtes semblent avoir été considérées comme secondaires au sein de l'institution. Le savoir propre à rendre possible une telle entreprise n'avait pas encore été établi. Aucun travail scientifique conséquent, aucun traité ne paraît avoir été consacré à ces « troupeaux ». L'assiette du projet de ménagerie n'avait pas été clairement fixée entre l'expérimentation, le soin, l'observation et l'ostentation. Plus sûrement, c'est l'absence d'un « berger » qui condamna le projet. Claude Bourgelat, hippiatre, ainsi que ses plus proches collaborateurs, ne purent jouer ce rôle. Ils étaient trop occupés à construire connaissances et réputation autour du cheval. Des considérations financières achevèrent de vider la ménagerie de ses animaux.

Un premier tournant eut lieu en 1783. Il appartient d'abord à l'histoire de la constitution de la médecine vétérinaire. Il se rattache ensuite à une histoire culturelle et politique plus large donnant son sens à un projet vétérinaire scientifique. Une seconde ménagerie fut en effet instituée sous Bertier de Sauvigny ${ }^{9}$. Un élevage «modèle » de bêtes à laine fut constitué à partir de quatre béliers et de neuf brebis. Cette recréation peut être lue de deux manières. D'abord dans ce qu'on a pu caractériser comme le moment «académique » de l'École vétérinaire d'Alfort. Des chaires d' « économie rurale », d' « anatomie comparée » et de « chimie » furent confiées respectivement à Louis-Jean-Marie Daubenton (assisté de Pierre- Marie-Auguste Broussonet), de Félix Vicq d'Azyr et d'Antoine-François Fourcroy ${ }^{10}$. La seconde lecture la replace dans le cadre d'une maturation du projet de développement économique porté par les milieux agronomiques. Ces deux mouvements puisant à quelques sources communes se rassemblaient dans la figure du berger.

De nombreux travaux l'ont bien montré, la médecine comme l'histoire naturelle ou l'agriculture jouèrent un rôle central dans l'élaboration d'un nouveau langage politique et d'une approche des possibilités offertes

(8) Philibert CHABERT, Pierre FLANDRIN, Jean-Baptiste HUZARD, Instructions et observations sur les maladies des animaux domestiques, 1791, nouvelle édition, Paris , imprimerie et librairie vétérinaire de J.B. Huzard, an III, p. 26.

(9) Sur le cadre de ces efforts agronomiques : André-Jean BouRDE. Agronomes et agronomies au XVIII siècle, Paris, S.E.V.P.E.N., 1967.

(10) Les trois titulaires des chaires étaient tous membres de l'Académie royale des Sciences. 
à l'action humaine ${ }^{11}$. Il s'agissait d'agir sur le milieu pour transformer et améliorer les conditions de vie des populations. Daubenton voulut fonder son enseignement à Alfort sur la conviction que « les animaux domestiques sont d'une très grande importance pour le maintien de la société et [...] pour le bonheur des hommes ». Ce processus de changement n'était pas qu'une métaphore : il renvoyait dans les parcs de l'école vétérinaire à un ensemble concret d'expérimentations et d'observations des rapports complexes entre la nature et l'animal, entre le sauvage et le domestique, entre la société et le milieu. Le vieux berger distinguait alors deux parties dans l'art vétérinaire : le gouvernement en santé des animaux et le traitement des maladies. C'est dans la première partie qu'il comptait fonder «l'économie vétérinaire ». Cette «science» devait « exposer les moyens de maintenir les animaux domestiques dans les bonnes qualités qu'ils ont acquises par nos soins et [...] faire des tentatives pour rendre ces animaux encore plus utiles qu'ils ne l'ont été jusqu'à présent ». Le moment académique fut aussi un moment naturaliste du champ vétérinaire par le biais de ce projet centré autour des catégories de régénération et de civilisation. Élèves et professeurs d'Alfort y furent associés et on eut dès la fin des années 1780 la formation de trois figures de « berger». D'abord Louis-Jean-Marie Daubenton qui œuvra à " soumettre à l'état de domesticité des espèces d'animaux sauvages dont nous puissions tirer du service et de l'utilité ». Il considérait alors de nombreux animaux exotiques ou sauvages et visait à offrir à la société des bêtes plus « sociables » et moins pétulantes, de la graisse, de la chair et du lait ${ }^{12}$. Pierre Flandrin (1752-1796) ensuite, neveu du directeur de l'école, un des premiers élèves de l'école lyonnaise, plus anatomiste et hippiatre que spécialiste des brebis et autres moutons, participa à ce projet. Dès 1785, il fut envoyé par le gouvernement en Angleterre puis en Espagne pour observer, étudier et décrire l'éducation des bêtes à laine. De ces voyages, il rapporta un certain nombre d'animaux plus ou moins exotiques, un goût affirmé pour l'économie rurale et la riche matière d'un traité souvent réimprimé. Ces connaissances lui permirent de s'affirmer au sein de la Société Royale d'Agriculture dont il fut correspondant. À partir de ses observations, il appelait à une entreprise plus large, à une « histoire actuelle de l'agriculture du globe » qui composerait en même temps « un cours complet sur cette

(11) Emma SPARY, Le jardin d'Utopie. L'histoire naturelle en France de l'Ancien Régime à la Révolution, Paris, Publications scientifiques du Muséum d'histoire naturelle, 2005.

(12) AN, F 10 1194, « Mémoire sur l'économie rurale vétérinaire et rurale par M. Daubenton » manuscrit de 16 pages. 
science $»^{13}$. Le troisième berger fut François-Hilaire Gilbert, élève tout à la fois d'Alfort et de Daubenton, esprit fin et travailleur infatigable, lecteur avisé des philosophes et vétérinaire admiré, et qui fut l'auteur célébré de traités sur les prairies artificielles. D'autres bergers œuvraient ailleurs. Au premier rang de ceux - ci on trouvait Jean- Marie Roland de la Platière, qui consacra aux bêtes à laine de nombreux développements dans ses volumes sur les « arts et manufactures » de l'Encyclopédie méthodique ${ }^{14}$. Si les références aux travaux d'Alfort furent bien rares, ceux-ci n'en étant qu'à leurs débuts, les bergers vétérinaires ne lui étaient pas inconnus ${ }^{15}$. Dans les écrits de ces bergers les animaux devenaient un des moteurs des transformations profondes qu'ils appelaient de leurs vœux.

Cette figure du berger permet de relativiser un certain nombre de coupures trop souvent opérées dans l'historiographie, en premier lieu entre le domaine vétérinaire, souvent renvoyé dans le monde des arts, et les sciences naturalistes, entre une école issue de la maréchalerie et des académiciens plus ou moins bien acceptés. Les bergers construisaient alors en discours mais également en actes, par leur activité et leurs troupeaux, un système qui n'était pas seulement scientifique ou technique mais qui fondait une véritable représentation de la société. Le «berger» Gilbert notamment proposait dès les dernières années de la monarchie absolue un autre mode de légitimation de la science fondée sur l'utilité, non seulement de l'animal mais encore plus largement du domaine vétérinaire, dissociant franchement cette utilité de la noblesse. La noblesse s'appliquant d'abord au seul cheval et par extension à son cavalier voire à celui qui le soignait, l'utilité la débordait, incorporait les bêtes à laine comme celles à cornes et séparait définitivement le champ vétérinaire de l'hippiatrie ou de la maréchalerie, son art auxiliaire.

« L'avantage le plus précieux dont puisse jouir une profession celui qui est le plus capable de toucher, de séduire, de captiver une âme délicate et sensible c'est sans contredit, Mrs, celui qui résulte de son utilité. Le sentiment secret des services qu'il rend à l'homme sera toujours pour l'homme même la source du plus pur de ses plaisirs, le seul moyen qu'il ait

(13) Pierre FLANDRIN, « Observations sur les moutons d'Angleterre », Mémoires d'agriculture, d'économie rurale et domestique publiés par la Société royale d'agriculture de Paris, Année 1789, trimestre d'été, Paris, Bureau de la Feuille du Cultivateur, 1789.

(14) Encyclopédie méthodique...op. cit.

(15) La correspondance de Madame Roland révèle qu'elle connaissait les travaux de Daubenton et de Broussonet, Lettres de madame Roland, Tome premier, 1780 -1787, Paris, Imprimerie nationale, 1900, p. 371. 
d'arriver au bonheur auquel il ne cesse d'aspirer. [...] On me dira peut être que la noblesse d'un état dépend surtout de celle des objets sur lesquels il s'exerce. Je n'examinerai point ici la force de cette objection, il ne me sera pas difficile de prouver que par la noblesse des objets qui l'occupent ; la médecine vétérinaire ne le cède à aucune autre profession. [...] Mais pourquoi chercher à prouver la noblesse des animaux, n'est ce donc pas l'homme lui-même qui est l'objet des travaux de l'artiste vétérinaire, n'est ce pas de la conservation de l'espèce humaine qu'il s'occupe en travaillant à celle de l'espèce animale ${ }^{16}$ ?

Dans ce glissement dans l'ordre du monde, le scientifique, le vétérinaire, ne travaillait plus seulement sur le possible - la naturalisation ou l'acclimatation, «l'économie vétérinaire » pour reprendre le lexique de Daubenton - mais surtout sur le réel, le déjà présent, c'est-à-dire qu'il consacrait sa vie à chercher dans l'étude de ces animaux les moyens de les conserver, de les multiplier, de prévenir leurs maladies et de les guérir, de faire disparaître les difformités dont le temps avait vicié les aspects, et d'augmenter leurs forces et par conséquent leurs services pour la société toute entière. Bien avant de prétendre à une dimension éthique comme on le voudrait aujourd'hui, l'élevage comportait une dimension morale. Celle-ci ne naissait pas de la nature même des animaux, ceux-ci ne devenant les serviteurs des hommes que par l' " effet de la contrainte qui leur a été imposée par la nature ». Elle n'existait que par ces nombreuses « obligations » de soin ou d'attention que ne pouvaient négliger que des hommes « ingrats » et « bornés ${ }^{17}$.

\section{Le berger, au cour d'un projet républicain}

Il semble ainsi possible de considérer qu' autour des « bergers » un projet avait vu le jour, prolongeant en direction de l'animal d'élevage un foisonnement intellectuel né dans les jardins et dans les ménageries. Ce projet devint « politique » en ce sens qu'à mesure que l'on avançait dans le siècle, les dynamiques politiques se chargeaient d'enjeux politiques et sociaux. La nature « politique » de l'œuvre du berger se lit particulièrement lors de deux séquences importantes formant comme des tournants dans le cours de la Révolution, de l'an II à l'an IV puis dans les mois qui suivirent le coup d'État du 18 Brumaire an VIII. C'est qu'à partir de 1789, le projet 
et la figure du « berger » s'enrichirent tout à la fois de la reconfiguration du champ des savoirs et des institutions scientifiques, de la promotion du savant en tant que remplissant une fonction sociale, des urgences et des nécessités des questions agraires et enfin de l'intégration de l'animal au cœur d'une dynamique industrielle.

\section{La reconfiguration du champ des savoirs}

Le plus célèbre des bergers d'Alfort, Daubenton, quitta l'école dès 1788 et ce départ marqua une rupture qu'il convient de ne pas négliger et qu'il ne faut pas lire à la seule lumière de l'histoire vétérinaire. Les animaux de la ménagerie le suivirent et l'école vétérinaire perdit ses dernières bêtes à laine. Si la fin de la présence du savant à Alfort fut d'abord associée à la question des économies et subsidiairement à la question de la délimitation du champ vétérinaire ${ }^{18}$, on peut y lire également comme la cristallisation ou l'accélération d'un processus marquant une mise à distance - réelle et critique - d'une figure du savant et d'un certain encyclopédisme, caractéristique des Lumières et l'achèvement d'une époque classique ${ }^{19}$. Les années 1789-1794 virent en effet une double dynamique, interne (au champ des bêtes à laine), et externe, tendant à la spécialisation. Interne si on considère la trajectoire des trois bergers que nous retrouvons désormais dans trois institutions animalières différentes ; Daubenton au Muséum d'Histoire naturelle, Gilbert au sein des domaines ruraux de Sceaux et de Rambouillet où des troupeaux consacrés aux expériences sur les bêtes à laine avaient été institués, l'un autour des moutons de race espagnole, l'autre autour des métissages ; Pierre Flandrin demeurait seul, déployant toute son activité au service de l'école d'Alfort. Une dynamique externe ensuite, si on considère la multiplication des lettres, mémoires et autres observations concernant les écoles vétérinaires. Une bonne vingtaine, tous pouvant être perçus comme une volonté de reconfigurer à des échelles et des degrés divers le champ vétérinaire alors en construction. Globalement absente des cahiers de doléances de 1789, la question de l'enseignement et des institutions vétérinaires n'en agitait pas moins différents cercles. Nous y retrouvons, outre la nécessité tellement déterminante de réformer les finances, la

(18) Pour justifier la réduction du nombre des chaires à Alfort, condition d'une contraction des dépenses de personnel, il fut décidé que désormais les professeurs ne pourraient être élus qu'au concours et parmi les élèves.

(19) Patrice BRET et Jean-Luc CHAPPEY, «Spécialisation vs encyclopédisme ? », La Révolution française [En ligne], 2 | 2012, mis en ligne le 15 septembre 2012, consulté le 19 septembre 2012. URL : http:///rf.revues.org/515 p. 3 et 4. 
question de l'autonomie de l'art vétérinaire par rapport à l'hippiatrie, celle de l'intérêt d'inclure la formation et la pratique vétérinaires dans le cadre plus large de l'histoire naturelle ou encore la question de l'autonomie de champ par rapport à la médecine. Il était donc question d'une manière de bornage du champ vétérinaire. Les interrogations portaient alors sur une « géographie » des sciences et des savoirs, y compris dans ses caractères les plus fondamentalement spatiaux au travers de projets de déménagement total ou partiel de l'École, à Paris ou à Versailles. Poser la question de la localisation, du site et de la situation, revenait en effet à envisager l'insertion ou l'absorption dans d'autres institutions ou dans certains corps de métiers ${ }^{20}$.

Toutefois la dynamique qui avait conduit Daubenton au Muséum d'Histoire naturelle et Gilbert à Sceaux, pouvant paraître affaiblir l'École d'Alfort, fut la même que celle qui permit de repousser les projets de rassemblement de différentes institutions médicales, naturalistes et vétérinaires en une seule entité parisienne et « encyclopédique ». De tels projets avaient notamment été présentés par Jacques-Henri-Bernardin de Saint-Pierre et surtout Broussonet qui fut, à Alfort, l'assistant de Daubenton. Le déclenchement de la Révolution et l'effervescence qui s'ensuivit participèrent ainsi d'une spécialisation accélérée des savoirs scientifiques. Ce mouvement de fond n'allait toutefois pas sans son balancement naturel, à savoir la recherche des moyens de reconstituer l'espace savant de la « république » naturaliste et de se raccrocher aux reliefs des logiques de patronage. Pierre Flandrin nous en fournit l'exemple. Jusqu'à la disparition des sociétés savantes, on le voit investissant ces cadres anciens comme moyen d'affirmer son inscription dans le monde savant. Membre correspondant de la Société d'Agriculture, il y présenta encore en 1793 un mémoire sur la rage. En ventôse an II, il était admis au sein de la Société d'histoire naturelle après lecture de deux mémoires d'anatomie ${ }^{21}$. C'est que, comme pour l'ensemble des savants naturalistes, l'enthousiasme avait fait place aux incertitudes et les vétérinaires d'Alfort, maréchaux ou bergers, souvent en position de défense, devaient maintenir des positions et des certitudes fraîchement acquises dans l'organisation des

(20) Par exemple Philippe Étienne LAFOSSE, Mémoire sur l'Ecole royale vétérinaire d'Alfort, Paris, Imprimerie L. Potier, 1789 ; Pierre Marie Auguste BROUSSONET, Réflexions sur les avantages qui résulteroient de la réunion de la Société royale d'agriculture, de l'Ecole vétérinaire et de trois chaires du Collège royal au Jardin du Roi. Paris, Imprimerie du Journal Gratuit, 1790.

(21) Jean- Luc CHAPPEY, Des naturalistes en Révolution, Paris, CTHS sciences, 2009, p. 234 
savoirs et des sciences. Ils s'efforçaient de trouver les moyens de poursuivre leur projet savant.

À partir de l'an III, cette dynamique d'atomisation devint l'une des dimensions d'une nouvelle configuration permettant de satisfaire les besoins professionnels de la communauté scientifique, besoins envisagés dans toute leur profondeur. Ce mouvement se caractérisait en effet par un renforcement institutionnel des lieux où les arts et les savoirs se définissaient et se précisaient et par l'apparition de lieux et de cercles offrant aux champs des savoirs une forme d'unité. Nos bergers se retrouvaient, échangeaient à l'intérieur des sociétés savantes qui se reconstituèrent sous le Directoire. Au printemps de l'an VI eut lieu la (re) fondation de la Société d'Agriculture du département de la Seine pour concourir aux progrès de toutes les branches de l'économie rurale ${ }^{22}$. Ce mouvement aboutit également à l'organisation de l'Institut national par la loi du 5 fructidor an III (22 août 1795). On retrouvait dans les cénacles de sa Première classe : Louis-Jean-Marie Daubenton, membre résidant, pour l'anatomie et la zoologie ; Pierre Flandrin, nommé mais qui mourut dans l'année, et François-Hilaire Gilbert. Là, les bergers côtoyaient d'autres savants naturalistes, botanistes ou vétérinaires tels qu'André Thouin, Henri Alexandre Tessier, Jacques Henri Cels, Antoine Augustin Parmentier ou Jean Baptiste Huzard ${ }^{23}$. L'entrée dans l'Institut ne couronnait pas seulement un savoir et un prestige, elle relevait également de l'incarnation et de la construction d'un projet non seulement scientifique mais avant tout politique, «le scientifique caractérisait l'image préférée de la société post-thermidorienne $»^{24}$.

Dans le domaine vétérinaire, le berger devenait celui qui incarnait le mieux et le plus la volonté d'explorer les processus de conservation et d'amélioration d'abord physique, ensuite morale et enfin politique. Rien d'étonnant donc à ce que Tessier, Gilbert, Daubenton et Flandrin, des savants dont l'œuvre semblait attachée aux bêtes à laine, se soient trouvés distingués par la République. Le projet qui fut celui des naturalistes sous l'Ancien Régime ${ }^{25}$ se diffractait au sein de l'Institut et d'une multiplication des lieux de « recherches » et de « développement » où la science «dure »

(22) Dominique Margairaz, François de Neufchâteau. Biographie intellectuelle, Paris, Publications de la Sorbonne, 2005, p. 435. an IV (1796)

(23) Institut national de la République française, Paris, Imprimerie de la République, Prairial

(24) Roger HaHN, L'anatomie d'une institution scientifique. L'Académie des Sciences de Paris, 1666- 1803, Paris, Édition des archives contemporaines, 1993, p. 430.

(25) Emma SPARY, Le Jardin d'Utopie...op. cit. 
se faisait et irriguait les lieux d'enseignement ${ }^{26}$. La disparition du troupeau d'expérience à Alfort, le primat du cheval dans les bâtiments et dans les cours et le refus de l'organisation des enseignements autour des différents animaux domestiques ne signifiaient alors nullement un recul de la figure du berger. Ce dernier œuvrait dans de multiples cercles, dont certains étaient incorporés à l’État.

\section{Une fonction sociale au sein du nouvel espace politique}

L'histoire de la construction de l'art vétérinaire et de sa transformation en une véritable discipline scientifique ainsi que la valorisation de la figure du berger s'inscrivaient en un rapport très étroit avec la construction de services administratifs aux compétences bien délimitées et bien définies et par une relative stabilité des personnels. En effet, par-delà les vicissitudes des réorganisations ministérielles, un double mouvement de définition de domaines de compétences et de technicisation de l'administration s'opérait ${ }^{27}$. Les bureaux du Comité d'agriculture, sous la Convention, puis du ministère de l'Intérieur s'étoffèrent et se spécialisèrent. Après la suppression du Conseil exécutif provisoire et des six ministres le composant, les écoles vétérinaires dépendirent d'abord de la Commission d'agriculture et des arts définie par décret des 12-13 germinal an II (1er et 2 avril 1794) ${ }^{28}$. Cette commission composée de deux membres et d'un adjoint avait de larges attributions parmi lesquelles on pouvait relever l'économie rurale, l'éducation des animaux domestiques et les écoles vétérinaires. Le 21 nivôse an II, les services furent réorganisés dans le sens d'un renforcement de la spécialisation. La commission fut scindée en cinq divisions qui prenaient désormais pour base les objets traités et non plus la nature du travail à effectuer. Sous le Directoire, les écoles vétérinaires étaient du ressort du ministère de l'Intérieur. Elles dépendaient de la quatrième division en charge de l'exécution des lois en rapport avec l'économie nationale dans les domaines de l'agriculture, du commerce, des manufactures. Cette division comprenait trois sections spécialisées composées à chaque fois d'un bureau consultatif et d'un bureau d'exécution. Une de ces divisions avait pour attribution l'agriculture. L'administration de l'agriculture se

(26) Gilbert par exemple maintint toujours un lien étroit avec l'École d'Alfort.

(27) Catherine KAWA, Les employés du ministère de l'Intérieur pendant la première République (1792-1800) approche prosopographique de la bureaucratie révolutionnaire, Thèse de doctorat de l'Université Paris 1, juin 1993 p. 68 - 70.

(28) Recueil des lois, décrets, ordonnances, Volume 5 annoté par M. Lepec, Paris, Administration du journal des notaires et des avocats, 1839, p. 226 - 227. 
trouva aussi confortée par une relative stabilité de la direction des divisions et des bureaux. Ces derniers devenaient des organes permanents assurant une continuité dans l'encadrement non seulement des écoles vétérinaires mais d'un champ très vaste.

Le mouvement de spécialisation n'était pas le fait unique des services de l'État. Cette première dynamique des Comités ou du ministère vers les institutions agricoles ou animalières se doublait d'un mouvement en retour des écoles et des bergers vers le ministère par l'intégration dans la sphère administrative d'un échelon scientifique et technique. Les comités, et par eux, plus largement et profondément la République, avaient en effet besoin d'experts pour traiter de nombreuses questions ayant à voir tant avec les urgences et les contingences les plus immédiates et les plus prosaïques qu'avec des projets engageant la prospérité nationale.

Trois des vétérinaires de l'École d'Alfort intégrèrent ces échelons dont deux de nos bergers : Pierre Flandrin et François-Hilaire Gilbert. S'y ajoutait Jean-Baptiste Huzard, un de leur plus prestigieux collègues. Pierre Flandrin intégra le Conseil d'Agriculture ${ }^{29}$ lorsque le second fut appelé par le pouvoir à siéger en 1794 en tant qu'agent de la troisième division (ou division de l'économie animale) de la Commission d'agriculture et des arts. Les Archives départementales du Val de Marne détiennent des fonds nous permettant d'envisager l'ampleur des travaux des bergers au sein des commissions du ministère. Nous y trouvons quelques mémoires ou correspondances soumis au regard de l'agent: des propositions de services de remèdes ou d'inventions couvrant une multitude de domaines, comme celle d'un procédé devant permettre de transformer les excréments humains en engrais sous forme de poudres inodores et faciles à transporter ; l'examen des moyens de lutter contre des menaces en rapport avec les animaux, bref, différents dossiers ayant à voir avec l'agriculture, pour l'essentiel, et le commerce. Si les domaines sont pléthoriques, on constate tout de même une spécialisation et la naissance d'une expertise : Gilbert s'attachait principalement aux diverses questions tenant à l'élevage et en particulier à celui des bêtes à laine : la médecine des ovins, l'introduction du mérinos, l'amélioration des laines en France ou la gestion des troupeaux de Rambouillet lui fournirent matière à réflexion.

La seule question de la lutte et de la prévention des délits ruraux révèle combien l'expertise de nos bergers était nécessaire à la République.

(29) Les siècles littéraires de la France ou nouveau dictionnaire historique, critique et biographique de tous les écrivains, morts et vivants, jusqu'à la fin du XVIII ${ }^{e}$ siècle, Tome $3^{\mathrm{e}}$, Paris, Desessarts, an VIII, 1800, p. 61-62. 
Les «méfaits agricoles » avaient connu une hausse tendancielle depuis les années 1780 et singulièrement depuis que le complexe seigneurial avait été entamé puis aboli. Les élevages, et en particulier celui des bêtes à laine, se trouvaient être la cause de nombreux délits. Les animaux lâchés sans conduite ni surveillance entraient dans les champs, condamnant l'ensemencement, entamant les récoltes et piétinant les jeunes pousses. Les bêtes errantes ou les troupeaux divagants réveillaient sans cesse la crainte ou la réalité des épizooties ${ }^{30}$. La question des troupeaux et de leur garde fut donc largement étudiée dans le cadre légal du Code rural comme dans celui des efforts agronomiques. Elle n'était pas seulement un débat économique ou « policier ». Il en allait en effet des principes de liberté et de propriété, d'une manière d'envisager les rapports sociaux et économiques, certains diraient qu'il en allait des rapports de production. Le troupeau séparé ou commun, enclos ou transhumant, devenait question politique, d'autant plus que dans ces aspects les plus immédiats, il fallait apaiser les tensions sociales et permettre à la masse des paysans de sortir de la pénurie. Les questions étaient complexes : la bête domestique était potentiellement une menace mais, même devenue nuisible, demeurait une nécessité. Ainsi la chèvre pouvait devenir calamité ou « vache $»^{31}$.

\section{Le berger et les «problèmes paysans de la révolution française »}

Si la propriété foncière était une question agronomique avant 1789, elle était assurément devenue un enjeu politique après cette date, et quel enjeu ! Ce qui était un problème fondamental des sociétés d'Ancien Régime, l'était plus encore pour un peuple en révolution ${ }^{32}$. Le berger offre alors une expertise pouvant constituer un pont entre les « agriculteurs de cabinet » et les " économistes », entre une masse de propriétaires et les savants parisiens, entre les problématiques agronomiques et les enjeux agraires. Autour de la question de la propriété, il devenait la figure non seulement de la modération, de la protection des domaines mais encore d'une possibilité de conjuguer les aspirations productives et les réalités sociales. Par son action, comme par ses écrits, il pouvait alors devenir porteur d'un projet,

(30) Octave FESTY, Les délits ruraux et leur répression sous la Révolution et le Consulat. Étude d'histoire économique, Paris, Librairie Marcel Rivière et Cie, 1956.

(31) On la désignait souvent comme la vache du pauvre. Animal destructeur dont les propriétaires bénéficiaient pourtant d'une large tolérance.

(32) Geneviève GAVIGNAUD - FonTAINE, Propriété et société rurale en Europe. Les doctrines à l'épreuve de l'histoire sociale française (1780 - 1920), Nantes, Éditions du temps, 2005 ; Albert SoBoul, les Problèmes paysans de la Révolution 1789 - 1848, Paris, Maspero, 1976. 
celui d'une République apaisée, confiante dans l'œuvre de ses savants pour agir sur la société.

La première dimension, celle de l'œuvre personnelle et directe, fut reprise par la postérité la plus immédiate, on en trouve trace par exemple dans les éloges posthumes. Le berger avait combattu pour le respect des exploitations et des troupeaux, pour la propriété, particulière comme nationale, pour la sienne comme pour celle d'autres savants. Ces actes ne pouvaient rester sans écho dans un pays où les propriétaires en appelaient régulièrement aux autorités pour faire respecter leurs droits. En effet, «Le vertige de la destruction qui signala l'ignorance parvenue à un pouvoir éphémère au moment du cataclysme de notre ordre social avait débordé tout ce que la raison humaine était capable de lui opposer. La hache de la démolition menaçait tous [les] monuments, [les] châteaux, [les] palais », Gilbert et son collègue Huzard usèrent de leur influence, de leur autorité et de leur entregent afin de préserver les établissements ruraux, au nombre desquels les domaines de Versailles, Sceaux et Rambouillet, riches de leurs bêtes à laine. Les mêmes vétérinaires furent également contraints de devenir les bergers d'un troupeau de Croissy près de Chatou. Un de leurs collègues de la commission d'agriculture ayant été contraint de fuir précipitamment laissant son troupeau menacé, les «bergers » prirent toutes les mesures afin d'assurer la conservation et la prospérité d'un précieux ensemble de bêtes métisses ${ }^{33}$. Par cette action de protection des biens, par ce refus de sacrifier le progrès aux tumultes et aux émotions, ces savants participaient à une lutte politique si fondamentale que l'animal n'en pouvait être absent. Le député de la Charente-Inférieure, Giraud, ne négligeant pas d'évoquer Gilbert dans ses citations, insistait en effet en l'an II pour que la République gardât quelques troupeaux de bêtes à laine entre ses mains. C'est que les animaux qui les composaient devenaient précieux et il ne fallait pas les laisser dégénérer :

«Autrement nous retomberions, pour cette branche, dans la barbarie dont nous faisons de si grands efforts pour nous tirer». Désormais, il « [pouvait] paraître piquant aux yeux du philosophe de voir les républicains français, qui pour jamais [avaient] anéanti la noblesse désastreuse qui

(33) M.L. BOUCHARD, Notice historique sur J. B. Huzard, extrait des annales de l'agriculture française (janvier 1839), Paris, Imprimerie de Mme Veuve Huzard, 1839 ; Georges CuvIER, Notice historique sur Hilaire-François Gilbert lue à la séance publique de l'Institut national le 15 vendémiaire an 10, Paris, Baudoin, Fructidor an X. 
existait parmi les hommes, si rigides observateurs de l'utile noblesse des moutons $»^{34}$.

Les bergers avaient déjà pris la plume autour de la question des bêtes à laine pour nourrir la pensée agronomique. Il fallait désormais l'utiliser pour assurer la prospérité des campagnes et le bonheur de leurs laborieux habitants, en tenant compte de toutes les transformations que la Révolution avait engendrées. Flandrin retiré puis décédé (1796), Daubenton tout à ses travaux au Muséum d'Histoire naturelle et à ses cours à l'École normale, c'est Gilbert qui s'en préoccupa le plus immédiatement. « Rien en agriculture ne doit être forcé pas même le bien, écrivait-il dans une adresse aux propriétaires de petites exploitations. Mais c'est un mal plus grand encore que de renverser une institution utile, par la seule raison qu'elle était née sous le règne de la tyrannie. » Il proposait un biais pour concilier ce qui était souvent présenté comme inconciliable, pour sortir de l'opposition entre l'agronome cultivateur savant et le paysan routinier et misérable. La France ne nourrissait qu'une partie des animaux nécessaires à ses travaux agricoles, à ses relations commerciales, à sa consommation, les épizooties ôtaient régulièrement et abruptement le quart, le tiers ou la moitié des troupeaux. Tout cela, Gilbert ne l'ignorait pas. Comme il n'était pas sans savoir que des propriétaires regardaient comme dangereuse la multiplication des troupeaux depuis 1789 et appelaient une action coercitive pour défendre les haies et les récoltes, ou bien encore qu'il était communément admis que seules les exploitations les plus grandes permettaient d'entretenir des troupeaux assez nombreux pour payer les frais d'un berger et qu'à l'inverse, dans les pays de petite culture, les bêtes étaient mal gardées, mal nourries et souvent attaquées par les mortalités. Loin d'ignorer ces constats, il s'en nourrissait. Mais il n'appelait pas pour autant à la réunion d'exploitations trop divisées, pas plus qu'aux mesures répressives promptes à réveiller des « passions haineuses ». Le problème, ce n'était pour lui, en l'an IV, ni le non-respect des lois, ni le partage des communaux pas plus d'ailleurs que leur défrichement ; le problème c'était surtout le mauvais usage qui était fait des portions défrichées. On s'était empressé de les cultiver en blé. Le berger appelait non pas à un retour en arrière, nécessairement brusqué, mais à une accélération, celle de la diffusion des progrès agronomiques, en particulier de la diffusion des

(34) « Réflexions sur la nécessité et la possibilité d'améliorer les laines en France, par MA.A. Giraud, député de la Charente inférieure à la Convention » compte-rendu dans La décade philosophique littéraire et politique par une société de républicains, Tome second, Paris, an II, p. 401. 
prairies artificielles ${ }^{35}$. De la contribution fondamentale du savant justifiant la liberté économique, de l'application des nouvelles techniques et des découvertes agronomiques devaient ainsi venir ces progrès essentiels qui stabiliseraient la société et permettraient la prospérité. Ces convictions, Gilbert les partageait alors largement avec les rédacteurs de la Décade philosophique qui lui ouvrirent dès lors régulièrement leur publication ${ }^{36}$.

Chez François-Hilaire Gilbert, alors le plus actif de nos bergers sur la glèbe et dans les bergeries, le républicanisme était donc né de l'articulation de la prise en compte de l'expérience révolutionnaire, y compris en ce qu'elle échappait aux savants - les questions agraires, en premier lieu, l'explosion de la violence - d'un programme social visant et engageant la formation d'un peuple éclairé et la promotion de principes humanitaires. C'est à partir de ces trois dimensions du républicanisme du berger qu'il faut opérer une réintégration de l'animal dans les questions sociales et politiques $^{37}$. Dès lors, l'engagement des « bergers » dans la lutte pour le maintien puis le développement des projets sociaux et économiques de la République pouvait les amener à apparaître comme des gestionnaires et des médecins compétents non seulement pour les questions agraires mais encore pour encadrer la société. C'est que ces savants participaient de la diffusion de la conviction exprimée dès les débats de l'an III par un des proches de François-Hilaire Gilbert que

«[...] le premier remède à nos maux, le premier principe de toutes les améliorations dont nous soyons susceptibles, consiste à tout faire pour l'Agriculture, parce que l'Agriculture peut seule vivifier toutes les branches de l'industrie nationale, parce que seule elle peut nous rendre les richesses que notre situation nous promet, la force qui en est le résultat, et les mœurs qui sont la sauve-garde des Républiques $»^{38}$.

Rien d'étonnant alors à ce que François-Hilaire Gilbert pût accéder au Corps législatif en 1799, achevant de construire une figure d'homme complet.

(35) La décade philosophique, 1795, tome 7 (vendémiaire - frimaire an IV), Paris, Bureau de la Décade an IV, p. $462-470$.

(36) Joanna KITCHIN, La Décade (1794-1807) un journal philosophique, MJ Minard, Lettres modernes, 1965 p. 145.

(37) Pierre SERNA, « Droits d'humanité, droits d'animalité à la fin du $18^{\mathrm{e}}$ siècle, ou la matrice du "racisme social" en controverse », Dix- Huitième siècle, n 42, 2010 ; Id., « The Rights of Man and the Rights of Animality, op. cit.

(38) Jean-Baptiste DUBOIS DE JANCIGNY, Vues générales sur l'amélioration de l'agriculture en France présentées à la Commission d'agriculture et des arts, Paris, Imprimerie de la Feuille du Cultivateur, an III, p. 17 - 18. 
Cet engagement politique explique l'inquiétude, d'abord, les formes de résistance, ensuite, qui agitèrent le milieu des bergers d'Alfort après le 18 Brumaire. S'il fallait en profondeur « révolutionner l'agriculture », la nécessité était désormais à la consolidation de tout ce qui avait été entrepris. Le coup d'État se conjuguait alors à la lutte et aux intrigues qu'avait fait naître le remplacement à l'Institut national de Daubenton, décédé le 21 décembre 1799. Déjà tourmenté par les difficultés du voyage qu'il effectuait en Espagne au nom du gouvernement pour extraire des bêtes à laine, accablé notamment par la faiblesse et l'irrégularité des subsides que la République lui expédiait, Gilbert reçut régulièrement des nouvelles constituant des menaces pour ce que ses amis et lui-même s'étaient efforcés de construire. C'est qu'avant même le 18 Brumaire, rien n'était fondamentalement acquis. Les domaines ruraux étaient fragilisés par les vues du ministre des Finances concernant Rambouillet et Versailles. Il fallait préserver les troupeaux des « vilains loups ». Après le coup d'État, les craintes semblèrent se réaliser. Il fallait désormais lutter « contre la fiscalité, contre [le] ministre lui-même et contre la faiblesse ». Les ralliements à Bonaparte devaient trouver des contreparties et les domaines en faisaient partie. Dès janvier 1800, la situation s'aggrava pour les établissements ruraux. Des éleveurs et des manufacturiers moins préoccupés de la pureté des races espagnoles que de profits rapides paraissaient avoir gagné l'oreille du ministre. Il fallait convaincre l'autorité de l'utilité de la dépense que les troupeaux représentaient pour l'État. Les amis de Gilbert s'efforcèrent de multiplier les arguments en faveur de leur œuvre. Ils jouèrent la carte alimentaire. L'intérêt de la viande de mouton mérinos fut mis en avant. Ils offrirent d'abord une bête de la race espagnole engraissée à Rambouillet au ministre qui la partagea avec les Consuls. Ils organisèrent ensuite un piquenique chez un restaurateur en vue de convaincre les bouchers parisiens et les autorités. Des quarante « amis » espérés, seuls vingt-huit vinrent déguster la viande, parmi lesquels Cuvier, Hallé, Parmentier, Dubois, Vilmorin, Rougier de la Bergerie, Silvestre, Coquebert. Malgré les défections, le dîner fut présenté comme une réussite, au moins sur le plan « gastronomique ». Plus généralement, Gilbert et ses amis jouèrent de la profondeur de leurs réseaux, en usant de tous les registres et de toutes les sociabilités, de l'Institut à la Société d'agriculture, du Moniteur à la Décade philosophique ${ }^{39}$. Hélas, le 6 mai 1800, Gilbert apprenait que les bergers n'avaient plus l'oreille du ministre, que le ministère avait été réorganisé et que désormais on 
allait au Conseil d'Agriculture comme on allait au café, désemparé et sans ouvrage. À l'été 1800 , le troupeau de bêtes métisses fut transféré dans l'école vétérinaire d'Alfort, le domaine de Versailles venant récompenser le sénateur Sieyès de ses engagements. Le 8 septembre 1800, François Hilaire Gilbert disparaissait en Espagne, mort de «besoin et de fatigue dans un village ignoré de Castille $»^{40}$.

\section{Vieux professeur, jeunes sociétés et nouveaux enjeux}

1799 et 1800 avaient vu les décès de Daubenton et de Gilbert ainsi que la disparition du domaine de Versailles. Pour autant, si cette période marque un tournant important dans l'organisation de l'espace savant vétérinaire, en particulier en l'orientant vers la création d'une organisation impériale des savoirs, la rupture de la dynamique qui faisait des bêtes à laine un moteur des transformations économiques et sociales ne fut que momentanée. Dès la fin de 1800, même en l'absence des « bergers » historiques d'Alfort, il y eut une reformulation dans une perspective quelque peu nouvelle, désormais industrielle.

Le vieux directeur de l'École vétérinaire, Philibert Chabert, eut conscience parmi les premiers de ce réinvestissement. Il avait senti la centralité de la question des bêtes à laine dans la réorganisation du champ vétérinaire et de l'économie rurale. En frimaire an IX, il sut opportunément profiter des changements à la tête du ministère de l'Intérieur. Jean-Antoine Chaptal ayant succédé à Lucien Bonaparte, les vétérinaires d'Alfort pensèrent retrouver écoute et possibilité d'influence. Dans une longue lettre, le directeur, homme de la maréchalerie, appelait à développer l'école vétérinaire d'Alfort autour des bêtes à laine. Tout à son objet, il débutait par un large et étonnant éloge du mouton :

« De tous les animaux domestiques sans même excepter le cheval, c'est le mouton qui est le plus utile comme aussi le plus multiplié. Il nous revêt de sa toison, il éternise sur sa peau nos conventions, il nous nourrit de sa chair, il adoucit nos mœurs par l'exemple des siennes, et ses intéressantes peuplades couvrent nos champs qu'elles sarclent et qu'elles engraissent. »

Philibert Chabert qui avait survécu depuis 1780 aux changements de régime et aux transformations institutionnelles avait su repérer le sens du courant et, hippiatre devenant berger, il offrait son autorité pour assurer

(40) Biographie universelle (Michaud), Tome 16, Nouvelle édition, Paris, chez madame Desplaces, 1854, p. 448. 
à un groupe décapité la continuité de l'œuvre et de l'enseignement de son confrère, désormais martyr, Gilbert. ${ }^{41}$ Cette initiative lui permit d'abord de réaffirmer la position centrale de l'École qu'il dirigeait dans le champ des institutions animalières et agricoles, ensuite et accessoirement, d'obtenir le transfert du troupeau de Versailles à Alfort. Une partie de l'œuvre de Gilbert, ses bêtes métisses, put ainsi être conservée. En quelques mois, les équilibres avaient été modifiés. Un retour sur un épisode antérieur l'éclaire. En brumaire an IX, Philibert Chabert avait déjà essayé de proposer une réforme de l'organisation de l'école d'Alfort. Ses arguments furent alors avant tout financiers. Ce fut un échec : même teintés de pédagogie, ils ne pouvaient justifier des transformations de l'enseignement vétérinaire que seules désormais la laine et les bêtes qui la portent vinrent permettre ${ }^{42}$. La même année, Augustin François Silvestre, proche de Chaptal et secrétaire de la Société d'Agriculture du département de la Seine, obtenait davantage d'écho puisque son Essai sur les moyens de perfectionner les arts économiques en France était notamment célébré par le Magazin encyclopédique de Aubin-Louis Millin de Grandmaison comme « une production vraiment patriotique ». Mettant en avant la nécessité de combattre les préjugés et les routines des cultivateurs français, il regardait « l'instruction publique comme le moyen le plus rapide et le plus assuré de faire faire des progrès aux arts économiques ; cette instruction doit agir sous plusieurs formes, être essentiellement théorique dans les écoles centrales, et pratique dans les fermes expérimentales et dans les écoles spéciales ». Parmi les six écoles qu'il prévoyait, une devait être consacrée à l'art vétérinaire, une autre à l'éducation des bêtes à laine et à leur préparation ${ }^{43}$. Une reprise des réflexions et des propositions autour du champ vétérinaire avait lieu à partir d'un projet industriel, auquel l'économie vétérinaire et l'économie rurale étaient désormais largement subordonnées.

Les Sociétés d'Agriculture puis, à partir de 1801, la Société d'Encouragement pour l'Industrie Nationale, devinrent d'importants relais pour la diffusion des idées tendant à construire une approche spécifique de l'agriculture tournée vers le développement des arts économiques au service de

(41) AN, F 10 1292, lettre de Philibert Chabert au ministre de l'Intérieur du 24 frimaire an IX (13 décembre 1800)

(42) AN, F 10 1279, « Tableau comparatif de la dépense des élèves de l'école vétérinaire d'Alfort en 1789 et en l'an VIII de la République avec celle proposée au ministère de l'Intérieur par le C. Chabert directeur de cette école » et lettre de Philibert Chabert au Ministre de l'Intérieur du 11 brumaire an IX (2 novembre 1800).

(43) Augustin François SILVESTRE, Essai sur les moyens de perfectionner les arts économiques en France; Imprimerie de Madame Huzard, an IX. 
l'industrie. Elles se renforcèrent et agirent comme des relais de l'État ${ }^{44}$. Le domaine des bêtes à laine fut largement investi au point que, voulant encourager les recherches et la valorisation des expériences, celles-ci multiplièrent les notices et les mémoires dans leurs bulletins, les concours et les distinctions. Les trois premières années d'édition des Bulletins de la Société d'Encouragement pour l'industrie nationale, on comptait treize articles traitant des ovins d'une manière large à propos d'expériences d'introduction ou de multiplication de nouvelles races ou de domaines plus précis comme du dessuintage des laines.

Aux côtés et parfois au-dessus de l'École d'Alfort, ces cercles s'arrogeaient progressivement le monopole de la « science utile » et contribuèrent à une mise en ordre des institutions scientifiques et agronomiques. Leurs membres siégeaient en surplomb de l'institution vétérinaire au sein d'une sorte de conseil tricéphale de surveillance des établissements d'agriculture, d'économie rurale et des pépinières et se retrouvaient également à l'intérieur du jury d'instruction des élèves d'Alfort. La laine était cause nationale et l'École d'Alfort participait de cet effort en travaillant dans quatre directions complémentaires formant un système cohérent de construction d'un véritable animal de rente, bien avant le milieu du siècle ${ }^{45}$. En premier lieu, le troupeau transféré à l'été 1800 depuis Versailles fut destiné à approfondir la maîtrise du choix des races et des opérations de métissage en vue de fournir à l'industrie nationale une matière première abondante, stable et variée. Ensuite, elle contribua à rendre possible l'accumulation du « capital sur pattes » en cherchant les moyens de permettre le rassemblement de troupeaux importants constitués de bêtes dont la valeur avait augmenté, en particulier par toutes les formes de recherches des moyens préventifs et curatifs des épizooties par la vaccination et l'hygiène. Alfort devait également être un « laboratoire » et un centre de formation où les savoirs et les techniques tenant à la nourriture de ces troupeaux seraient affirmés et affinés. Un cours d'économie rurale destiné aux agriculteurs fut créé. Enfin, à défaut de pouvoir former les propriétaires, une école des bergers vit le jour en l'an IX.

(44) Jean-Pierre ChaLINE, Sociabilité et érudition. Les sociétés savantes en France, Éditions du comité des travaux historiques et scientifiques, 1995 ; Jean-Luc CHAPPEY, « Héritages républicains et résistances à "l'organisation impériale des savoirs" », AHRF, n³46, p. 97 - 120 ; Id., La Société des observateurs de l'homme (1799-1804), Paris, Société des études robespierristes, 2002 ; Dominique MARGAIRAZ, François de Neufchâteau, op. cit.

(45) Nathalie JAS, « Déqualifier le paysan, introniser l'agronome, France 1840 - 1914 » Écologie et politique, Presses de Sciences Po, 2005/2, n³1, p. 45- 55. 


\section{L'attention revient sur les bergers}

À partir de 1800, l'attention était revenue sur les bêtes à laine. Les conditions agraires jugées les plus propices au progrès des élevages n'ayant pu être généralisées, il devint nécessaire de s'intéresser à ceux que l'absence d'enclosure rendait plus nécessaires : les bergers. Davantage qu'à la recherche des moyens de limiter les « délits ruraux » et la divagation du bétail, la création d'une École des bergers à l'intérieur de l'école vétérinaire répondit à un objectif de soin, de protection et de prospérité de troupeaux, de plus en plus précieux, dans des cadres ruraux qui n'avaient pu être profondément modifiés. En ces domaines, la question agraire dominait les aspects agronomiques. De prairies artificielles et de pâturages enclos, il ne pouvait pas encore être question. Si l'Anglais Ellis prétendait qu' « un berger boiteux et un chien paresseux [étaient] les plus propres à la garde d'un troupeau », le transfert d'une telle maxime demeurait impensable en France. Le modèle se dérobait et les savants et les praticiens durent se replonger dans l'œuvre de leurs devanciers. La formation des bergers était en effet une préoccupation ancienne et avait déjà une histoire en dehors des écoles vétérinaires. Dans les dernières années de l'Ancien Régime, l'abbé Tessier, Roland de la Platière et surtout Daubenton s'étaient efforcés de convaincre leurs contemporains de s'y consacrer ${ }^{46}$.

Au moins dans les discours, les vues de Daubenton dominaient et Jean-Baptiste Huzard s'empressait d'associer son nom à une nouvelle édition de l'Instruction du « berger de Montbard », publiée par ordre du Gouvernement ${ }^{47}$.

« Il faut [écrivait Daubenton] savoir plus de choses pour le métier de berger que pour la plupart des autres emplois de la campagne. Un bon Berger doit connaître la meilleure manière de loger son troupeau, de le nourrir, de l'abreuver, de le faire pâturer, de le traiter dans ses maladies, de l'améliorer, et de faire le lavage, et la tonte de la laine. Il doit savoir conduire son troupeau et le faire parquer, élever ses chiens, les gouverner et écarter les loups $»^{48}$.

(46) Encyclopédie méthodique... op. cit., et « article berger », Agriculture par l'abbé Tessier, et M. Thouin, Tome second, Paris, Panckoucke, 1791.

(47) Instruction pour les bergers et pour les propriétaires de troupeaux avec d'autres ouvrages sur les moutons et sur les laines par Daubenton, troisième édition, publiée par ordre du gouvernement, Paris, Imprimerie de la République, an X.

(48) Louis Jean Marie DAUBENTON Instruction pour les bergers et pour les propriétaires de troupeaux, Paris, imprimerie de Ph. D. Pierres, 1782, p. 3. 
Dans les campagnes pourtant, la formation des bergers, et plus inquiétant encore, l'attention qui leur était portée demeurèrent en dessous des enjeux économiques et sociaux. Un peu partout, on regrettait leur impéritie, leur inexpérience et leur inefficacité. Ici, le berger était figure de la superstition, là de la faiblesse face au loup. Encore en l'an III, dans les vues sur l'amélioration de l'agriculture en France qu'il présenta à la Commission d'agriculture et des arts, Jean-Baptiste Dubois de Jancigny rappelait que cette «classe d'hommes qui n'a jamais été mise à sa place parmi nous, est, en général, dépourvue de lumières et de moralité ». La « régénérer » était un des premiers pas à effectuer en vue d'obtenir une véritable amélioration des bêtes à laine. Il préconisait alors l'institution de "pépinières » dans les départements ${ }^{49}$. Certains, comme François-Hilaire Gilbert, avaient milité pour la constitution de grands troupeaux, seul moyen permettant d'offrir une rémunération intéressante et donc de permettre l'emploi de techniciens capables et instruits. Une Instruction pour les bergers, fut-elle rédigée par Daubenton, ne pouvait donc suffire. Au sein de la Société d'Agriculture de la Seine puis de la Société d'Encouragement pour l'Industrie nationale, les réflexions furent reprises. Philibert Chabert en profita pour réclamer la création d'une école spécialisée. Par commodité, conviction ou opportunisme il la voulait incluse dans son école. Ainsi se trouverait renforcée l'emprise de l'institution qu'il dirigeait sur le champ vétérinaire. Son argumentaire formait comme une synthèse des vues des milieux agronomiques. Il fallait former les « bons bergers » pouvant seconder efficacement des propriétaires souvent « accablés d'affaires » et en conséquence obligés de se reposer sur des individus spécialisés. Était-il possible d'acquérir ce talent ailleurs qu'à l'école d'Alfort ? Chabert le niait : Les vétérinaires dans les campagnes, trop peu nombreux, ne pouvaient ni ne voulaient offrir cette instruction et cet exemple par peur d'être dépossédés d'un savoir qu'ils voulaient être leur monopole et parce qu'ils n'étaient pas professeurs. Il écartait également les livres. C'est que d'abord il n'en existait pas de suffisamment bons à son idée et que les bergers, cela était bien connu, ne savaient pas lire. Quant à la transmission traditionnelle, se faisant à l'ombre de la bergerie par des parents, elle était à proscrire puisqu'elle n'ouvrait que sur la perpétuation des routines et des erreurs ${ }^{50}$.

(49) Jean Baptiste DuBOIS DE JANCIGNY, Vues générales sur l'amélioration de l'agriculture en France présentées à la Commission d'agriculture et des arts, Paris, imprimerie de la Feuille du Cultivateur an III, p. 17 - 18.

IX.

(50) AN, F 10 1292, lettre de Philibert Chabert au Ministre de l'Intérieur du 24 frimaire an 
Le ministre de l'Intérieur, Jean-Antoine Chaptal, ainsi qu'il le précisa dans un discours qu'il prononça lors de l'Assemblée générale de la Société d'encouragement pour l'industrie nationale qu'il présidait, reprit les vues de Silvestre et les encouragements de Philibert Chabert.

« [Croyant] que l'art de soigner les bêtes à laine étoit plus négligé parmi nous que celui qui a pour objet l'éducation, le traitement ou le régime de plusieurs autres animaux domestiques ; [il avait] pensé qu'un des premiers moyens d'améliorer cette partie de l'économie rurale, consistoit à confier la conduite des troupeaux à des hommes plus instruits que ceux qui en ont été chargés jusqu'ici : en conséquence, [il avait] appelé de divers points de la France et [avait] réuni à Alfort des bergers qui avoient conduits les troupeaux pendant plusieurs années; [il avait] demandé aux préfets des hommes jeunes encore, et par conséquent susceptibles d'instruction $»^{51}$.

La première année, les cours ne durèrent que six mois. Cette formation accélérée aurait donné d'excellents résultats selon le ministre. Les années suivantes la formation dura une année pleine, conformément aux vues exprimées par le directeur de l'École vétérinaire. Une durée plus importante était inutile. Ce laps de temps suffisait à voir et apprendre tout ce qu'il fallait et présentait l'avantage de pourvoir à une multiplication rapide des « bons bergers ». Le programme de cette année ressemblait en ramassé aux grandes articulations des études vétérinaires mais appliquées à un seul animal. La formation était divisée en trois parties. Lors des quatre premiers mois, les élèves devaient étudier l'anatomie élémentaire du mouton, les principes de physiologie et de physique. La période suivante voyait l'étude de l'hygiène, de l'histoire naturelle du mouton, des moyens d'améliorer les races, de la manière dont les troupeaux devaient être logés, nourris, parqués et soignés. Enfin, on donnait aux élèves-bergers des bases concernant le traitement des maladies, les opérations chirurgicales et la préparation des médicaments ${ }^{52}$. Une fois la formation achevée à Alfort, la plupart seraient allés suivre un perfectionnement à Rambouillet ${ }^{53}$.

Si les « excellents résultats » furent rapidement vantés, cette école ne subsista que quelques années. Une lettre que l'inspecteur des écoles

(51) Bulletin de la Société d'Encouragement pour l'Industrie nationale, Troisième année $\mathrm{n}^{\circ} 1$, Messidor an XII, Paris, Huzard Fuchs, an XII, p. 4.

(52) Charles DE LASTEYRIE, Histoire de l'Introduction des moutons à laine fine d'Espagne dans les divers Etats d'Europe et au Cap de Bonne Espérance, Paris, Levrault, an XI - 1802 p. 85 - 86. XIV.

(53) AN, F 10 1293, lettre de Jean Baptiste Huzard au Ministre de l'Intérieur du 9 nivôse an 
vétérinaires, Jean Baptiste Huzard, adressa en l'an XIV (décembre 1805) au ministre de l'Intérieur nous éclaire sur cette disparition.

«Cette institution qui pouvoit être très utile, qui [avait] produit quelques bons élèves et qu'un assez grand nombre de propriétaires [réclamaient] encore, étoit établie sur une base vicieuse dans la forme et dans le fond, elle n'a pu se soutenir, et il [avait] fallu y renoncer. Les élèves bergers payés plus chers que les élèves vétérinaires n' [avaient] pas tardé à se croire supérieurs à ceux-ci et ne [s'étaient] plus regardés comme des hommes destinés à devenir des bergers, mais bien à être des artistes bergers, propres à diriger des troupeaux et ceux qui les [conduisaient] en se promenant la badine sous le bras. Ils [étaient] devenus raisonneurs, libertins, je fus même obligé dans le temps, d'expulser de Rambouillet ceux de ces messieurs qui ne vouloient rien faire, et de placer à l'hospice des vénériens à Paris, ceux que le libertinage avoient rendus malades ; d'un autre côté, les élèves d'Alfort, voyant des bergers, dont l'instruction était payée plus chèrement que la leur, se décourageaient et il en résultait une comparaison cause de désagrément dans un établissement public $\aleph^{54}$.

Dans maints domaines, en particulier politiques, les mêmes causes ne produisent pas toujours les mêmes effets. C'est là une vérité assez commune dont il faut nous saisir afin de mettre en perspective et en question l'apparente permanence d'une dynamique autour des bêtes à laine. Le motif par lequel Jean Baptiste Dubois de Jancigny avait appelé, en l'an III, à instituer des écoles pour régénérer la « classe » des bergers, c'est-à-dire son absence de moralité, avait servi en l'an XIV à Jean Baptiste Huzard pour justifier la fermeture d'une de ces institutions. C'est qu'au-delà du maintien des stéréotypes peut se lire la transformation d'un projet nourri dans les jardins et les ménageries d'Alfort et de Paris, visant à une régénération de la société autour de l'histoire naturelle et, pour nous, des moutons en une dynamique industrielle de création d'un animal et, pourrait-on dire, d'un esprit de rente. À un discours appelant à intégrer un groupe social afin qu'il trouve «sa place » répondrait alors une forme de stigmatisation qui se trouve justifiée en réalité par des problèmes plus profonds tenant à l'économie et à la société, à la structure de la propriété comme à des limites médicales. Ce n'est pas sans raison que dans l'Histoire de la France rurale, la première des trois séquences d'un long $\mathrm{XIX}^{\mathrm{e}}$ siècle vient s'achever en 
$1852^{55}$ : si l'effet d'entraînement de l'« industrie agricole » existait, il était lent et se heurtait encore durant l'Empire à de nombreux murs.

La dynamique autour des bêtes à laine et des bergers pose également, pour nous, la question de la définition de la marge. Les animaux formaient alors une marge paradoxale. Ces animaux, on les retrouvait en effet partout, critères et reflets des distinctions et des hiérarchies sociales, agents de l'organisation de l'espace des sociétés. Dans le champ des savoirs, ils pouvaient également légitimer des positions et des découpages entre les différents domaines. Il n'est pas anodin de relever que le premier des huit points développés par Jean-Baptiste Huzard dans le document préparatoire qu'il transmit à Georges Cuvier pour ses fameux rapports à l'empereur était consacré aux seules bêtes à laine.

« Vous connaissez [écrivait-il] tout ce qui est relatif à l'introduction du mérinos en France, et à l'amélioration de nos laines. Cette révolution, dont l'origine est due à Trudaine et à Daubenton, a été effectuée complettement par cette commission d'agriculture qui a tant construit à une époque où on détruisait tout, et qui n'a fait que du bien, va empêcher le mal, au milieu des dévastations de tous genres $[\ldots] \gg^{56}$.

Leur centralité était bien perçue, au début du XIX ${ }^{\mathrm{e}}$ siècle, à l'intérieur comme au-delà du champ vétérinaire, tant par un fils de maréchal-ferrant, Jean Baptiste Huzard, que par Jean-Antoine Chaptal. Elle le fut sans doute moins chez les historiens ${ }^{57}$.

Malik MeLlaH

Doctorant

Institut d'Histoire de la Révolution française - EA 127 - CRHM Université Paris 1 Panthéon Sorbonne 17 rue de la Sorbonne 75005 Paris malikmellah@hotmail.fr

(55) Georges Duby et Henri Wallon (dir), Histoire de la France rurale, Tome 3 Apogée et crise de la civilisation paysanne de 1789 à nos jours, Paris, éditions du Seuil, 1976.

(56) Bibliothèque de l'Institut, MS 3139, Fonds Cuvier, rapport de Jean Baptiste Huzard à Georges Cuvier (18 mars 1808).

(57) Jean Marc MORICEAU, Histoire et géographie de l'élevage français Paris, Fayard, 2000. L'auteur notait une certaine occultation de l'élevage dans les ouvrages sur la « révolution agricole ». 